\title{
Proceso participativo de producir un mapa integrado de Centroamérica y sur de México
}

\author{
Marcela Norori* \\ Carren Williams, Elam Torres \\ Leonardo Salazar, Eduardo Sáncho \\ Emma Flores, Blanca Isabel Gómez, \\ Francisco Benítez, Regina Menendez, \\ Milton Núñez, Alex Martínez, \\ Jean Parcher, Roberto Lugo, \\ Eufracio Zavala, Noel Ramírez, \\ José Pérez, Carlos Morales, \\ Elizabeth Samuels, Rogelio Mondragón
}

\begin{abstract}
The development of a regional map based on the official geospatial datasets of the National Geography Institutes of Central America and Mexico is an essential element for the use of people who inhabit the region to aid in their everyday decision making process, both for community and regional scales. This Regional Map of Central America and southern Mexico, integrates elements relating to: politicaladministrative divisions of the regions - departments, states and provinces- major urban settlements, the roads, water bodies and rivers. The digital map can be used to determine the areas influences and contribute to the prevention and disaster mitigation to safeguard human lives and natural resources.

Utilizing the participatory mapping approach with support from the Pan American Institute of Geography and History (PAIGH) to integrate the geospatial data information between eight countries not only assures the continuity of the project, but also strengthens the relationship between the technical specialists in the region. This type of initiative provides a means to use maps to allow the countries be to able to capture the movements and trends of development of peoples.
\end{abstract}

* Dirección General de Catastro y Geografía de Honduras, Honduras, C.A., correo electrónico: marcela_norori@hotmail.com 
Key words: Central America, México, integrated map, transboundary, participatory.

\section{Resumen}

La elaboración de un mapa regional basado en los datos geoespaciales oficiales de los institutos geográficos de Centroamérica y México constituye un elemento fundamental para las personas que habitan la región para la toma de decisiones tanto a escala local como regional. El mapa regional de Centroamérica y sur de México, integra elementos relativos a: las divisiones político-administrativas de las regiones - departamentos, estados y provincias-, los principales asentamientos urbanos, las vías de comunicación, cuerpos y corrientes de agua; este mapa permite determinar las área de influencias y coadyuvar a la toma de medidas de prevención y mitigación de desastres para salvaguardar las vidas humanas y los recursos naturales.

Utilizando la metodología participativa y con apoyo del Instituto Panamericano de Geografía e Historia (IPGH), integrar la información geoespacial entre estos ocho países no solamente asegurará la continuidad de este proyecto, sino que también fortalece las relaciones entre los especialistas técnicos de la región. Este tipo de iniciativas deben ser un medio para que a través de los mapas los países puedan plasmar los movimientos y las tendencias de evolución de los pueblos.

Palabras clave: Centroamérica, México, mapa integrado, transfronterizas, participativo.

\section{Antecedentes}

El monitoreo del cambio climático a una escala global requiere la cooperación entre las naciones y para que esta cooperación se haga efectiva, las naciones necesitan compartir y hacer compatible sus datos ambientales, geográficos y demográficos, de tal manera que es necesario promover la colaboración entre naciones, sin que se comprometa la seguridad nacional. En Centroamérica, muchos de los Institutos Geográficos Nacionales han completado sus conjuntos de datos del Mapa Global, y están considerando aplicar los estándares y el contenido de los datos del Mapa Global, a los datos de alta resolución de sus países. En el año 2009 Jean Parcher, geógrafa del U.S. Geological Survey, preparó la primera solicitud al Instituto Panamericano de Geografía e Historia (IPGH) para iniciar los talleres participativos en Centroamérica, la propuesta prometía llevar a cabo unas series de reuniones de trabajo entre los representantes de los Institutos Geográficos Nacionales de todos los países de Centroamérica y de México, con el objeto de construir un mapa digital integrado de toda la región. El pequeño tamaño de la región Centroamericana vista en una escala mundial y el gran número de desastres naturales que afecta la región, hacia imprescindible contar con información geográfica para diversos usos. 
En los cuatro años que han transcurrido desde el 2009 hasta el 2013 se llevaron a cabo tres talleres para la integración de los datos geográficos de Centroamérica y Sur de México.

Existen algunos datos sobre desastres naturales provenientes de previas actividades de mitigación, junto con datos ambientales relacionados a proyectos, sin embargo los mismos no han estado integrados. Las actividades de mapeo integradas regionalmente son esenciales para garantizar la compatibilidad de los datos a largo plazo a través de la región.

\section{Objetivos}

Aplicar métodos de cartografía participativa para armonizar e integrar datos cartográficos digitales para la región Centroamericana y el sur de México en una escala de 1:250,000 o mayor.

\section{Desarrollo de talleres participativos}

\section{Primer taller}

El primer Taller Centroamericano de Integración de Datos y Capacitación Técnica se llevó a cabo en la ciudad de Heredia, Republica de Costa Rica entre los días 21 al 27 de agosto de 2011. Participantes provenientes de los institutos geográficos nacionales de los siete países centroamericanos, de México, y de los Estados Unidos se reunieron en la Universidad Nacional de Costa Rica con el propósito de armonizar las capas base de datos geoespaciales a través de las fronteras internacionales para crear un mapa digital continuo de Centroamérica.

Todos los participantes concurrieron con sus datos oficiales para trabajar a escala 1:250,000 de manera participativa, colaboraron con sus países vecinos para integrar los temas de hidrografía, vías, poblados y límites administrativos en un conjunto de datos regional. Este taller estuvo financiado por las Comisiones de Cartografía, Geografía, y Geofísica del Instituto Panamericano de Geografía e Historia (IPGH) y la empresa Enviromental Systems Research Institute (ESRI).

\section{Segundo taller}

El segundo Taller Centroamericano de Integración de Datos y Capacitación Técnica se llevó a cabo en la ciudad de Sonsonate, República de El Salvador, entre los días 28 de noviembre al 3 de diciembre de 2011 con el apoyo logístico y administrativo del Centro Nacional de Registros, Instituto Geográfico y del Catastro Nacional de El Salvador (CNR). El taller estuvo financiado por las Comisiones de Cartografía, Geografía, y Geofísica del Instituto Panamericano de Geografía e Historia (IPGH), el programa GeoSUR y la empresa Enviromental Systems Recearth Institute (ESRI). 
Durante este taller se le dio continuidad al primer taller con los mismos participantes provenientes de los institutos geográficos nacionales de los siete países de Centroamérica, de México, y de los Estados Unidos con el objeto de armonizar las capas base de datos geoespaciales nacionales para crear un mapa digital continuo de Centroamérica y el sur de México. Después de revisar la armonización de las cuatro capas básicas ( límites administrativos, hidrología, vías y poblados), los participantes se pusieron de acuerdo en los rasgos y atributos en común que se debían incluir en el mapa digital continuo de Centroamérica. Cada Institución Geográfica armonizó sus datos para integrarlos en el conjunto de datos regional, trabajando en conjunto con los países con los que compartían fronteras. Como conclusión a este taller se dispuso que este mapa integrado de la región debía estar disponible en la web, para lo cual el programa GeoSUR mostro interés en apoyar esta iniciativa.

\section{Tercer taller}

El tercer Taller Centroamericano de Integración de Datos y Capacitación Técnica también se llevó a cabo en la ciudad de Sonsonate, República de El Salvador, El Salvador entre los días 21 al 27 de abril de 2013 con el apoyo logístico y administrativo del Centro Nacional de Registros, Instituto Geográfica y del Catastro Nacional de El Salvador (CNR) (véase Figura 1). El taller estuvo financiado por las Comisiones de Cartografía, Geografía, y Geofísica del Instituto Panamericano de Geografía e Historia (IPGH), el programa GeoSUR y la empresa Enviromental Systems Research Institute (ESRI).

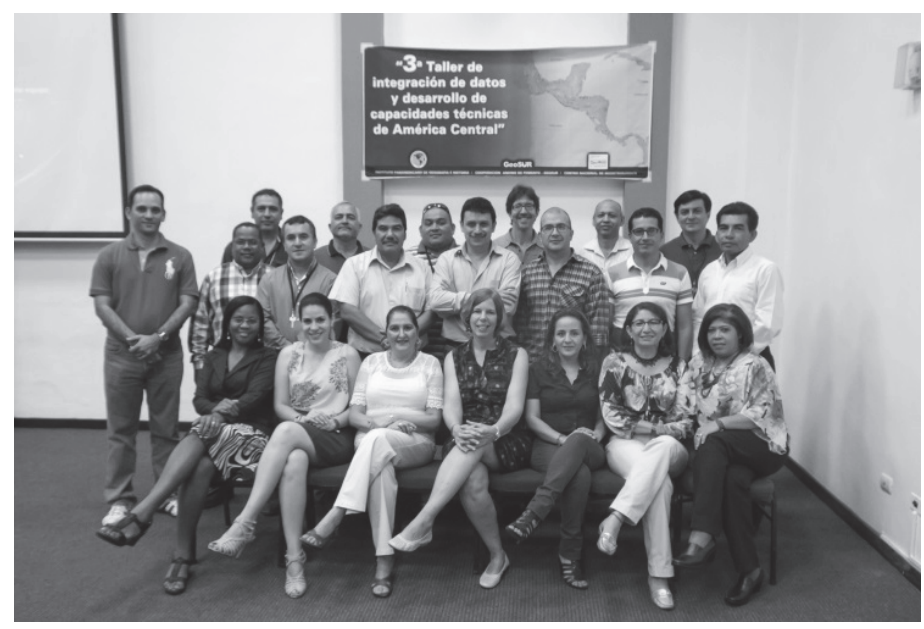

Figura 1. Participantes de Centroamérica, México y Estados Unidos durante el "Tercer Taller de Integración de Datos y Desarrollo de Capacidades Técnicas de América Central", llevado a cabo en El Salvador en abril del año 2013. 
En este taller se terminó con la integración de las capas principales, se decidió la simbología cartográfica a utilizar en el mapa digital integrado de Centroamérica y sur de México (Mapa Meso-Americano), también se hicieron los metadatos para cada capa. Al finalizar el taller se obtuvo el producto integrado de ocho capas de información: límites administrativos (límites nivel 1, límites nivel 2 y límites nivel 3), hidrografía (ríos, cuerpos de agua), vías (carretera internacional, carreteras principales y secundarias), poblados (mancha urbana y asentamientos).

\section{Metodología}

Se integraron ocho capas para el mapa de Centroamérica y sur de México, contenidas dentro los cuatro grupos siguientes:

I Tema de Límites Administrativos, compuesto de 3 capas: Nivel 1, Nivel 2 y Nivel 3.

II Tema de Hidrografía, compuesta de 2 capas: Ríos y Cuerpos de Agua.

III Tema de Poblados, compuesto de 2 capas: Poblados y Mancha Urbana.

IV Tema de Vías, compuesto de 1 capa: Vías.

En el tema de Límites Administrativos, la capa de Nivel 1 se refiere a la mayor división política en el país, como departamentos, provincias o estados. Luego seguido la capa del Nivel 2 son las divisiones políticas que le siguen dentro del Nivel 1 como los municipios o cantones. Por último el Nivel 3 se refiere a las divisiones más pequeñas del país tales como las aldeas en el caso particular de Honduras o el corregimiento en Panamá.

Al comienzo del primer taller los representantes de los distintos países presentaron su data y hubo un buen diálogo sobre los problemas que se tendrían que solucionar durante la integración de las capas. Las capas seleccionadas habían sido creadas a escala de 1:200,000 y otras a escala 1:25,000.

\section{Descripción general de la diferencia en la data}

Al iniciar la revisión de la data se pudo observar que los rasgos representativos entre las capas adyacentes en muchas zonas no coincidían con el rasgo pertinente al cruce de la frontera. Esto ocurre comúnmente en capas digitales y en particular capas que se digitalizan a escala de 1:200,000 o menor, y más si se trata de capas procedentes de distintas organizaciones. En otros casos fue visible la diferencia entre la densidad de los rasgos entre capas adyacentes. Por ejemplo en una capa representando los rasgos de los ríos, la cantidad de rasgos en un país en algunos casos eran mucho más densos a los rasgos de la capa del país vecino. Estas diferencias de densidad y alineamiento entre capas de países vecinos se trabajaron en conjunto con representantes de ambos países para llegar a un acuerdo en cuanto al cambio que se llevaría a cabo para integrar la data. 
Otro problema bastante complicado que se tuvo que enfrentar fue el de las diferencias entre los atributos y sus definiciones (Tabla 1).

Al momento en que se intentaba unir las capas las definiciones de los campos y el contenido de los atributos en sus respectivas tablas no combinaban correctamente, por lo que era imposible unirlas sin antes preparar las tablas creando nuevos campos y modificando la data.

De una manera participativa y después de un largo diálogo, con el propósito de obtener uniformidad en los campos de todas las capas contribuidas el grupo decidió que los campos tendrían ciertos nombres y tipos definidos para que así se les diera un despliegue y descripción uniforme a los rasgos y a su significado.

Tabla 1

Atributos de las capas trabajadas

\begin{tabular}{|c|c|c|}
\hline Capa & Campos & Ejemplos de data en campos respectivos \\
\hline \multirow{9}{*}{ Poblados } & País & MX, BE, GT, HN, SV, NI, CR, PA \\
\hline & Categoría & Departamento, Estado, Municipio, Provincia, \\
\hline & & Urbana, Rural, District, Town, City \\
\hline & Cod_Ctegoría & 1, 2, 3, 4 IE. Dept (1). Estado (1). District (1), \\
\hline & & City (1), Town (2), \\
\hline & & Municipio (2), Rural (2) \\
\hline & Nombre & \\
\hline & Población & \\
\hline & Cod_Unico & \\
\hline \multirow{3}{*}{ Mancha urbana } & País & MX, BE, GT, HN, SV, NI, CR, PA \\
\hline & Nombre & \\
\hline & Población & \\
\hline \multirow{6}{*}{$\begin{array}{l}\text { Límites } \\
\text { administrativos }\end{array}$} & País & $\mathrm{MX}, \mathrm{BE}, \mathrm{GT}, \mathrm{HN}, \mathrm{SV}, \mathrm{NI}, \mathrm{CR}, \mathrm{PA}$ \\
\hline & Categoría & Departamento, Estado, Municipio, Provincia, \\
\hline & Cod_Ctegoría & Urbana, District, Town, City \\
\hline & Nombre & 1, 2, 3, 4 IE. Dept (1), Estado (1), District (1), \\
\hline & Población & City (1), Town (2), Municipio (2) \\
\hline & Cod_Unico & \\
\hline \multirow{5}{*}{ Carreteras } & Nombre & \\
\hline & Tipo & Pavimentada. No Pavimentada, Otro \\
\hline & Nivel & Panamericana (1), Pavimentada (2), \\
\hline & País & No Pavimentada, Otro (3) \\
\hline & & MX, BE, GT, HN, SV, NI, CR, PA \\
\hline \multirow{2}{*}{ Ríos } & Nombre & \\
\hline & Tipo & MX, BE, GT, HN, SV, NI, CR, PA \\
\hline \multirow{4}{*}{ Cuerpos de agua } & Nombre & \\
\hline & Tipo & Perenne, Intermitente \\
\hline & Clasificación & Lago, Laguna, Embalse, Corriente de agua, \\
\hline & País & MX, BE, GT, HN, SV, NI, CR, PA \\
\hline
\end{tabular}


Por ejemplo en el caso de Poblados existía una variedad de campos diferentes para cada país y luego de la discusión el acuerdo fue mantener sólo seis campos para describir los poblados; los campos seleccionados fueron campos que estaban presentes en la mayoría de las capas de los países. Las otras siete capas fueron trabajadas de igual forma en referencia a los campos y atributos de las tablas.

\section{Integración de la data}

Inicialmente en el primer taller se integraron geográficamente las ocho capas, utilizando para ello imágenes Landsat que permitieran ver como se unían la data entre las fronteras de cada país; cada participante de un país integraba sus capas con las de los países vecinos y así sucesivamente. En el segundo y tercer taller, luego de que se definió el orden de los campos de todas las capas, los participantes prepararon sus capas añadiendo campos nuevos y completando los campos con los atributos apropiados. Cuando se terminó de verificar que la data tuviera los atributos correctos se procedió a unir todas las capas de los distintos temas en capas continuas y representativas de cada tema. El producto final resulto en ocho capas representando cuatro temas. Todas las capas se crearon y se manipularon utilizando el SIG de ESRI versión 9.3, 10.0 y 10.1 de ArcGIS. Todas las capas integradas se convirtieron a una geodatabase y se proyectaron a la proyección geográfica WGS1984.

Posteriormente estas capas se entregaron a los representantes del Centro Nacional de Registros de El Salvador, quienes asumieron el compromiso de preparar el producto final en digital y habilitarlo al público en un WMS en el servidor de GeoSUR.

\section{Metadatos}

En el marco del tercer taller se desarrolló la tarea de documentar las capas que se estuvieron trabajando desde el primer taller, a través de metadatos; éstos se gestionaron según el estándar ISO 19115, a través del perfil Lamp, utilizando la herramienta de ArcCatalog versión 10.1 (de ESRI). Este trabajo fue ejecutado por un grupo de técnicos de Guatemala, Belice, El Salvador, Costa Rica y Panamá, quienes identificaron, recolectaron y documentaron los campos establecidos como: obligatorios, condicionales y opcionales.

El proceso de captura se estableció documentando un archivo *.xml con los datos generales que sirvió de plantilla y luego se dividieron entre los integrantes del grupo de trabajo las tareas de: recopilación de la información, traducción del español al inglés y la documentación de los datos particulares de cada una de las capas. Como resultado se obtuvieron metadatos que permiten: la búsqueda, acceso, descripción y difusión de los datos, que han adquirido mayor valor. 


\section{Publicación del mapa}

La publicación estuvo a cargo de los representantes del Centro Nacional de Registros de El Salvador. Esta se hizo en el Geoportal de GeoSUR $<$ http://www.geosur.info/geosur/>, con la proyección WGS 1984 Web Mercator (auxiliary sphere), y se representó en las escalas que son comunes para otros servicios en línea como ESRI Online, Google Maps o Bing Maps. Por tal razón, el mapa tiene como escala mínima la de 1:9,244.649 y una escala máxima de 1:144,448. Así mismo, cabe destacar que la leyenda del mapa se elaboró tanto en español como en inglés, para lograr una mejor comprensión de todos los usuarios. Entre los elementos geográficos que el mapa representa están los Limites Administrativos, que por su naturaleza se representan a su vez en límites de primer nivel, límites de segundo nivel y algunos países como El Salvador y Honduras contribuyeron también con los límites de tercer nivel.

También se integró otros elementos geográficos, como los núcleos de población, que en el mapa se representaron en Núcleos de Población Principales y Núcleos de Población Menores, con el fin de que sean representados correctamente en las escalas que se definieron para el mapa y evitar así una saturación visual de elementos. Así mismo se representaron las áreas urbanas también conocidas como manchas urbanas, además también se incluyó las carreteras principales haciendo una clasificación de carreteras internacionales, carreteras primarias y caminos, siempre con el fin de que sean representados correctamente en la escala adecuada (Figura 2). De la misma forma se representó la hidrografía, que incluye dos capas geográficas, los cuerpos de agua y los ejes de los ríos principales. El mapa tiene como fondo la representación del relieve sombreado, conocido como hillshades o Shaded Relief, que se ha extraído del Modelo Digital de Elevación SRTM90, permitiendo agregar también la capa de Elevaciones que es representada por una rampa de color que permite diferenciar visualmente las diferentes alturas de la región. La combinación de estas dos capas permite al mapa tener un fondo adecuado para una visualización correcta de los elementos geográficos representados (Figura 3).

\section{Fuente de datos de cada país}

\section{México}

Los representantes del Instituto Nacional de Estadísticas y Geografía (INEGI), utilizaron la - Base Cartográfica a escala 1:250,000 del Proyecto Sistema Unificado de Información Nacional (SUNIL) del Instituto Geográfico Nacional (IGN).

También utilizaron datos de la base Cartográfica a escala 1:50,000 realizada en 2009, con fotografía aérea año 2006. Información que fue obtenida mediante procesos de generalización cartográfica a partir del mapa base escala 1:50,000 cuyo origen de este último fue por métodos fotogramétricos. 
Los archivos vectoriales de datos geográficos para la cartografía topográfica escala 1:250,000 están compuestos por elementos de planimetría y altimetría, y específicamente los datos usados para los talleres únicamente se trabajaron con datos de planimetría. Los datos de México se apegan a los estándares internacionales consistentes en que los datos representados deben cumplir la exactitud de $.2 \mathrm{~mm}$ por la escala del producto. Y las bases cartográficas de los datos fueron las siguientes:

\section{Guatemala}

Los representantes del Instituto Geográfico Nacional (IGN) utilizaron datos cuyo origen data de la cartografía digital obtenidos del Proyecto Sistema Unificado de Información Nacional (SUNIL) - que se generó en el Instituto Geográfico Nacional (IGN) en el año 2000, con la cooperación de varias instituciones del Gobierno de Guatemala. Dicho proyecto agrupó las capas de vías de comunicación, hidrografía, poblados, cabeceras municipales, límites departamentales y municipales, a una escala 1:250,000. Sin embargo habían transcurrido más de 10 años desde aquel proyecto hasta el año 2013, por lo que fue necesario hacer una actualización de la información, utilizando cartografía digital a escala 1:50,000 con fotografías aéreas del año 2006, para poder conformar el continuo de información con México, Belice, El Salvador y Honduras.

\section{Belice}

Los representantes del Centro de Información de Tierras (LIC) del Ministerio de Recursos Naturales y Agricultura (MNRA) de Belice contribuyeron con una data a una escala de 1:250,000 que también es producto de la generalización de una data original a escala 1:50,000.

\section{El Salvador}

Las representantes del Instituto Geográfico y del Catastro Nacional (CNR), que es una dependencia del Centro Nacional de Registros, incluyeron para la elaboración de este mapa integrado, los datos vectoriales de El Salvador que fueron elaborados como parte del proceso de edición cartográfica, con restitución fotogramétrica del periodo 2004-2008, empleando el programa de Microstation y ArcGIS. Esta data es precisamente con la que se elaboró el Mapa Oficial de la República de El Salvador a escala 1:200,000 con fecha de creación del año 2005 y con revisión en el año 2006. 


\section{Honduras}

En el caso de Honduras, la representante de la Dirección General de Catastro y Geografía del Instituto de la Propiedad (DGCG) utilizó los datos oficiales del país que son producto de una generalización de la cartografía básica en escala 1:50,000 y que fue elaborada a través de procesos fotogramétricos análogos por el antiguo Instituto Geográfico Nacional y que en el año 2000 fueron digitalizados; la capa de vías fue actualizada en el año 2010 por la Secretaría de Obras Públicas, Transporte y Vivienda, mientras que la capa de mancha urbana también fue actualizada en el año 2010.

\section{Nicaragua}

El representante del Instituto Nicaragüense de Estudios Territoriales (INETER) proporcionó datos de Nicaragua que fueron elaborados en el marco de la actualización de la cartografía básica en el periodo comprendido entre los años 2000-2002, data que también se obtuvo de la generalización a 1:250,000 de la base cartográfica con la que se elaboró el mapa básico del país a escala 1:50,000 e imágenes del satélite Spot de 1998.

\section{Costa Rica}

Los representantes del Instituto Geográfico Nacional (IGN) de Costa Rica proporcionaron información cartográfica que data del año 1968, a escala 1:50000, la misma fue producida en forma analógica mediante el proceso de separación de colores; utilizando las técnicas de gravado en láminas de película estable (scribe coat) y a partir de mosaicos de compilación derivados de la restitución fotogramétrica análoga. Esta base cartográfica fue elaborada por el Instituto Geográfico Nacional (IGN), Ministerio de Obras Públicas y Transportes, San José; y posteriormente fue generalizada a una escala de 1:200,000 y revisada en el año 1988. La proyección original es la Cónica Conforme de Lambert, utilizando el esferoide de Clarke de 1866 con el Datum terrestre "Fundamental de Ocotepeque". Las diferentes capas de información ríos, carreteras, etc., fueron digitalizadas en el 2006 y en el año del 2008 fueron reproyectadas al sistema de proyección CRTM05, que según el Decreto Ejecutivo No 33797-MJ-MOPT del 30 de marzo de 2007 se convierte en el sistema de coordenadas oficial para la República de Costa Rica. Esta proyección es una adaptación de la proyección Transversal de Mercator adaptada para Costa Rica.

En el caso de la información de Límites Administrativos, la escala de digitalización fue 1:50,000 a la cual se le sobrepuso el borde del país a escala 1:200,000. Esta capa fue actualizada en noviembre del 2012. Las capa de poblados y de mancha urbana fue levantada a escala 1:50,000 y digitalizadas en los años 2010 y 2011 respectivamente. 


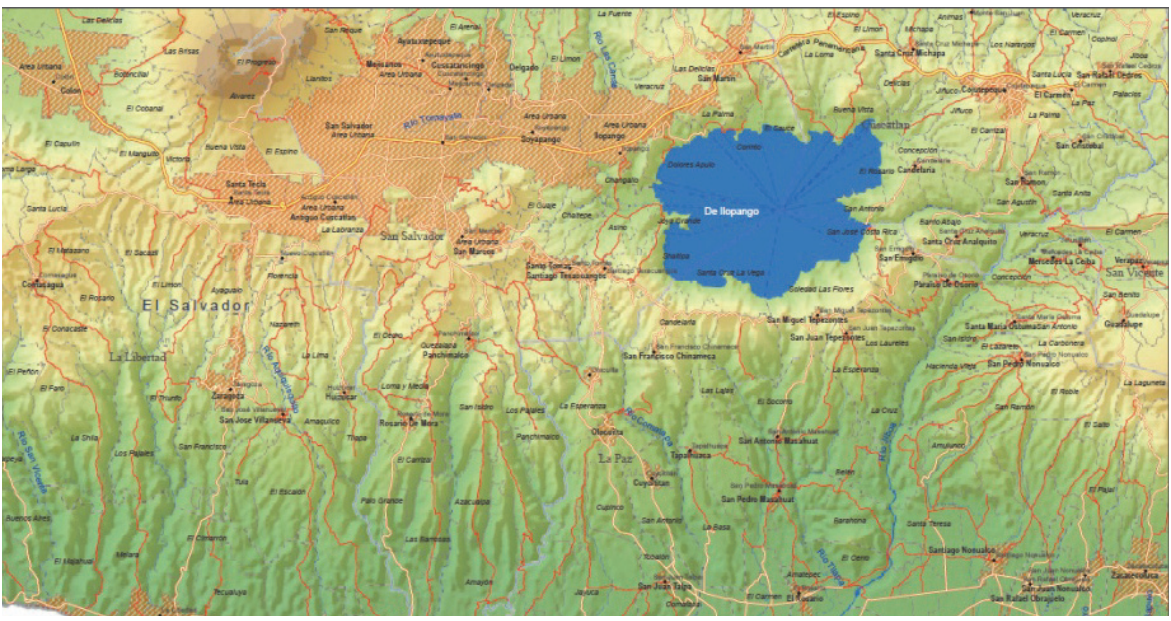

Figura 2. Representación de las áreas o manchas urbanas con las principales vías, en la imagen se observa al país de El Salvador .

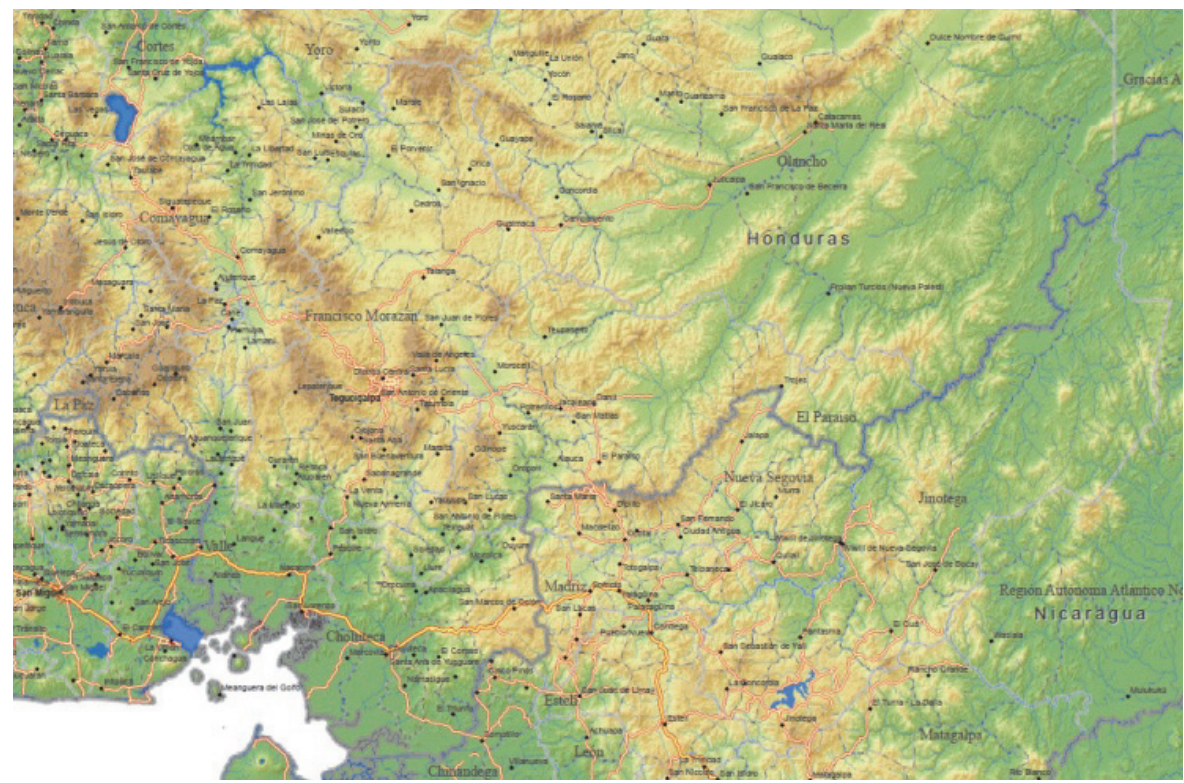

Figura 3. En la figura de puede observar el fondo del relieve sombreado, extraído del MDT SRTM90, que se utilizó como base para el Mapa Integrado de Centroamérica y Sur de México. 


\section{Panamá}

El representante del Instituto Geográfico Nacional "Tommy Guardia”, proporcionó datos de la base cartográfica para la cual fue creado el Mapa General de la República de Panamá, con una escala digital de 1:250,000. Las capas de hidrografía, manchas urbanas, masas de agua y red vial datan del año 2007 ; la capa de poblados data del año 2010 mientras que las capas de Límites fue actualizado en abril 2013.

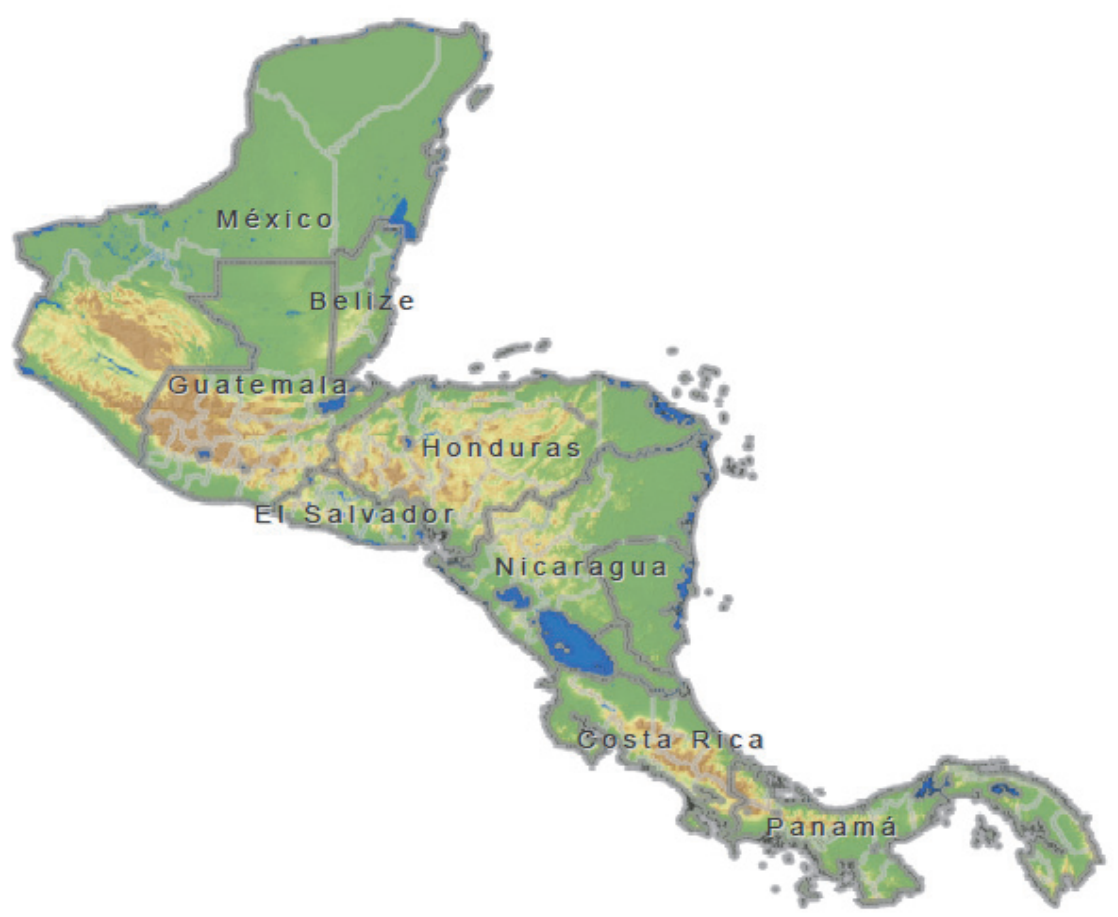

Figura 4. Resultado del Mapa Integrado de Centroamérica y Sur de México en producto digital listo para publicarse en el Geoportal de GeoSUR.

\section{Resultados}

1. Mapa de Integración de datos de Centroamérica y Sur de México y/o Mapa Mesoamericano, que reúne los elementos geográficos básicos de cada país: la parte sur de México, Guatemala, Belice, Honduras, El Salvador, Nicaragua, Costa Rica y Panamá.

2. Publicación del mapa en un ambiente web, que permite realizar ajustes que se han considerado necesarios, como una extracción uniforme de elementos, para 
evitar que unos países tengan una mayor saturación que otros en algunos elementos particulares como los núcleos de población o la hidrografía, además que también permite la integración de la red hidrográfica y de la misma forma la red vial.

\section{Conclusiones}

Se logró producir el mapa integrado de Centroamérica y sur de México, el cual ayudará a estudiar los fenómenos naturales en la región, localizando y orientando los fenómenos que se presenten. Además este mapa será útil para el planeamiento y la investigación urbano-regional ya que organiza la información espacial en una serie de capas básicas. Cada capa de información contiene datos con similares características y estructura gráfica entre los distintos países. Los datos de las diferentes capas comparten el mismo entorno geográfico, lo que permite la superposición de otro tipo de información de tipo raster y vectorial, para realizar análisis y otros estudios.

También puede tener aplicación para fomentar el turismo de la región ya que el mapa muestra las principales rutas de traslado entre los distintos lugares; y al encontrarse integradas esas rutas existe la posibilidad de realizar en forma sencilla el cálculo de distancias entre los lugares.

El mapa puede servir de base para plasmar los movimientos políticos, migratorios, económicos, las tendencias de los pueblos, la evolución, entre otros, con ello se puede entender cómo va evolucionando la población de esta región a través del tiempo.

Un logro intangible logrado a través de este proyecto fue la oportunidad de crear y fortalecer las relaciones entre distintos especialistas técnicos de la región, siendo este un paso crucial para alcanzar la cooperación, con fundamento técnico, entre las naciones, lo cual facilita la dinámica de compartir datos ante la ocurrencia de contingencias naturales o antrópicas o bien ante la implementación de programas de desarrollo regional.

\section{Siguientes pasos}

Es necesario seguir construyendo conjuntos de datos geoespaciales continuos y con estructuras similares para toda Centroamérica, por lo cual en el tercer taller, se definió que el siguiente paso será continuar armonizando y estructurando las siguientes capas temáticas regionales:

- Curvas de nivel

- Redes hidrográficas (para integrarlas y conectarlas) y

- Cuencas hidrográficas 
Ya que uno de los usos más importantes del mapa regional es incorporar el uso de las herramientas SIG que permitan reducir los riesgos de desastres naturales ante la ocurrencia de fenómenos hidrometeorológicos extremos, y el monitoreo de los ecosistemas locales y regionales ante la amenaza del cambio climático, con el fin de ayudarnos a preparar datos geoespaciales útiles para desarrollar modelos de monitoreo y de predicción para la región que den apoyo a los gobiernos en la implementación y desarrollo de programas de prevención o bien programas y acciones que busquen minimizar riesgos, vulnerabilidad, implementación de programas de desarrollo, fundamentado en el manejo integral de cuencas, particularmente ante los efectos del cambio climático y el deterioro del medio ambiente, en búsqueda de que la región para lograr el desarrollo sostenible.

\section{Participantes del "Taller de integración de datos y desarrollo de capacidades técnicas de América Central" 20 al 27 de abril, 2013, Sonsonate, EI Salvador}

\begin{tabular}{|c|c|c|c|}
\hline País & Representante & Correo electrónico & Institución \\
\hline Belice & $\begin{array}{l}\text { Carren Williams } \\
\text { Elam Torres }\end{array}$ & $\begin{array}{l}\text { plio@mnrei.gov.bz } \\
\text { elamtorres@yahoo.com } \\
\text { plio@mnrei.gov.bz }\end{array}$ & $\begin{array}{l}\text { Land Information Center } \\
\text { Land Information Center }\end{array}$ \\
\hline Costa Rica & $\begin{array}{l}\text { Leonardo Salazar } \\
\text { Eduardo Sáncho }\end{array}$ & $\begin{array}{l}\text { lsalazar@rnp.go.cr } \\
\text { leosalazar2010@gmail.com } \\
\text { esancho@rnp.go.cr } \\
\text { eduardo.sancho@gmail.com }\end{array}$ & $\begin{array}{l}\text { IGN de Costa Rica } \\
\text { Registro Nacional } \\
\text { IGN de Costa Rica } \\
\text { Registro Nacional }\end{array}$ \\
\hline El Salvador & Emma Flores & dflores@cnr.gob.sv & $\begin{array}{l}\text { Instituto Geográfico y Catastro } \\
\text { Nacional }\end{array}$ \\
\hline & Blanca Isabel Gómez & $\begin{array}{l}\text { bgomez@cnr.gob.sv } \\
\text { isabelloso@yahoo.com }\end{array}$ & $\begin{array}{l}\text { Instituto Geográfico y } \\
\text { Catastro Nacional }\end{array}$ \\
\hline & Francisco Benítez & $\begin{array}{l}\text { fbenitez@cnr.gob.sv } \\
\text { frankb.photos@gmail.com }\end{array}$ & $\begin{array}{l}\text { Instituto Geografico y Catastro } \\
\text { Nacional }\end{array}$ \\
\hline EUA & $\begin{array}{l}\text { Jean Parcher } \\
\text { Robert Lugo }\end{array}$ & $\begin{array}{l}\text { Jean_parcher@ios.doi.gov } \\
\text { rlugo@usgs.gov }\end{array}$ & $\begin{array}{l}\text { Department of the Interior } \\
\text { United States Geological Survey }\end{array}$ \\
\hline Guatemala & $\begin{array}{l}\text { Regina Menendez } \\
\text { Milton Núñez }\end{array}$ & $\begin{array}{l}\text { regedu2004@gmail.com } \\
\text { xmasdos@gmail.com } \\
\text { milton.nunez@ign.gob.gt }\end{array}$ & IGN de Guatemala \\
\hline Honduras & Marcela Norori & marcela_norori@hotmail.com & $\begin{array}{l}\text { Dirección General de Catastro y } \\
\text { Geografía, Honduras }\end{array}$ \\
\hline & Alex Martínez & Martinezalex776@gmail.com & $\begin{array}{l}\text { Dirección General de Catastro y } \\
\text { Geografía, Honduras }\end{array}$ \\
\hline México & Rogelio Mondragón & $\begin{array}{l}\text { Rogelio.Mondragon@inegi.org.mx } \\
\text { rogeliomb@yahoo.com }\end{array}$ & INEGI \\
\hline Nicaragua & $\begin{array}{l}\text { Eufracio Zavala } \\
\text { Noel Ramírez }\end{array}$ & $\begin{array}{l}\text { Eufracio.Zavala@inegi.org.mx } \\
\text { noel.ramirez@gc.ineter.gob.ni }\end{array}$ & INEGI \\
\hline & José Pérez & $\begin{array}{l}\text { nramirezro@gmail.com } \\
\text { josefpe2005@gmail.com } \\
\text { francisco.perez@gc.ineter.gob.ni }\end{array}$ & $\begin{array}{l}\text { INETER } \\
\text { INETER }\end{array}$ \\
\hline Panamá & $\begin{array}{l}\text { Carlos Morales } \\
\text { Elizabeth Samuels }\end{array}$ & $\begin{array}{l}\text { cmorales@anati.gob.pa } \\
\text { carlosmorales822@gmail.com } \\
\text { esamuels@anati.gob.pa } \\
\text { lilysamuels@gmail.com }\end{array}$ & $\begin{array}{l}\text { IGN "Tommy Guardia" } \\
\text { IGN "Tommy Guardia" }\end{array}$ \\
\hline
\end{tabular}

